\title{
Demonstration of the Common Antigens of Clostridium botulinum, $C$. sporogenes and $C$. novyi by an Enzyme-linked Immunosorbent Assay and Electroblot Transfer
}

\author{
By IAN R. POXTON \\ Bacteriology Department, University of Edinburgh Medical School, Teviot Place, \\ Edinburgh EH8 $9 A G, U K$
}

(Received 19 October 1983)

EDTA extracts were prepared from whole cells of 16 strains of Clostridium botulinum (types AE), 6 strains of $C$. novyi (types A-D) and 3 strains of $C$. sporogenes. They were reacted in an enzyme-linked immunosorbent assay (ELISA) with antisera raised against whole, UV-killed cells of $C$. sporogenes and $C$. novyi type A. Results showed significant cross-reactions between $C$. sporogenes antiserum and the $C$. botulinum type A (three out of four strains), proteolytic type B (all strains) and one type $\mathrm{E}$ strain, and between $C$. novyi type $\mathrm{A}$ antiserum and $C$. botulinum types $\mathrm{C}$ and $\mathrm{D}$. All the $C$. sporogenes and $C$. novyi strains reacted with their homologous antiserum; these two species showed no cross-reactions. All the reactions were investigated further by running the EDTA extracts on SDS-polyacrylamide gels. The separated molecules were electrophoretically transferred to nitrocellulose membranes, reacted with antiserum and complexes visualized with horseradish peroxidase conjugate reagents. Only those extracts that reacted significantly in the ELISA gave a pattern of cross-reactive antigen bands and the number of bands and intensity of stain closely paralleled the strength of the ELISA reaction.

\section{INTRODUCTION}

The heterogeneous group of clostridia collectively known as Clostridium botulinum are alike in that they all produce toxins with a similar pharmacological action. In taxonomic terms, however, strains of $C$. botulinum can be divided into four reasonably distinct groups: Group I, proteolytic strains with serologically distinct A and B toxins; Group II, non-proteolytic strains producing toxins of types B, E and F; Group III, strains producing toxins of types C and D; and Group IV, proteolytic, non-saccharolytic, toxigenic type G strains (Smith, 1977). In culture and biochemistry, excluding toxin production, Group I strains are indistinguishable from $C$. sporogenes; accordingly $C$. sporogenes is considered by some workers to be a non-toxigenic variant of $C$. botulinum (Iida et al., 1981). Various degrees of cross-reactivity between the two species have been revealed by immunofluorescence procedures (Walker \& Batty, 1964; Hatheway \& McCroskey, 1981) and by numerical taxonomy; for example, Kiritani et al. (1973) grouped 20 proteolytic strains of $C$. botulinum and 14 strains of $C$. sporogenes in the same phenon. Similarly Group III C. botulinum strains (i.e. types $C$ and D) are indistinguishable from $C$. novyi. Type $C$ C. botulinum can be cured of the prophage that codes for toxin production and converted by another phage to a $C$. novyi type A strain, and $C$. botulinum types $\mathrm{C}$ and $\mathrm{D}$ can be interconverted by phage (Eklund \& Poysky, 1981).

Our extensive study of cross-reactive antigens in the genus Clostridium (Poxton \& Byrne, 1984) demonstrated shared antigens between $C$. botulinum types $\mathrm{A}$ and $\mathrm{B}$ (proteolytic) and $C$. sporogenes. Moreover, antigens from types $\mathrm{C}$ and $\mathrm{D}$ cross-reacted with antiserum to $C$. novyi type A. The present study extends the investigation of these cross reactions.

Abbreviations: $\mathrm{npB}$, type B non-proteolytic strain; $\mathrm{pB}$, type B proteolytic strain; TBS, Tris-buffered saline. 


\section{METHODS}

Bacteria. All strains of $C$. botulinum were from the collection in the Botulinum Laboratory, Unilever Research, Sharnbrook, Bedford, UK : type A, strain nos 1, 7, 8, 9; type B (proteolytic, pB), strain nos 25, 26, 27; type B (nonproteolytic, npB), strain nos $188,194,229$; type C, strain no. 205; type D, strain no. 154 and type E, strain nos 34 , $118,126,250$. The $C$. novyi strains NCTC 538 (type A), NCTC 9747 (type C) and NCTC 8350 (type D) and $C$. sporogenes NCTC 8594 were obtained from the National Collection of Type Cultures, Central Public Health Laboratory, Colindale Avenue, London NW9 5HT, UK. Other strains from our laboratory culture collection were: C. novyi GR2A and GR3A (type A strains) and GR2B (type B), and C. sporogenes strains 24 and 33 . The strains of $C$. botulinum were checked for toxin type by $\mathrm{Mr}$. Grimes of Unilever Research at the time of EDTA antigen preparation. The culture supernates were used in mouse toxin-antitoxin neutralization tests as outlined by Willis (1977). Neutralizing antitoxin was obtained from the Pasteur Institute, Paris, France. The identity of all strains was confirmed. The strains of $C$. novyi and $C$. sporogenes were fully characterized by the method of Holdeman et al. (1977).

Growth conditions. Stationary phase bacteria $(0.2 \mathrm{ml})$ which had been cultured in Robertson's cooked meat broth and incubated anaerobically with $10 \% \mathrm{CO}_{2}$ for up to $48 \mathrm{~h}$ at $37{ }^{\circ} \mathrm{C}$, were used to inoculate $100 \mathrm{ml}$ proteose peptone/yeast extract broth (Holbrook et al., 1977). Cultures were incubated anaerobically with $10 \% \mathrm{CO}_{2}$ for $16 \mathrm{~h}$, or $40 \mathrm{~h}$ for $\mathrm{C}$. botulinum types $\mathrm{C}$ and $\mathrm{D}$, at $37^{\circ} \mathrm{C}$. Spore formation as judged by phase-contrast microscopy was negligible under these conditions.

Antigen extraction. Extraction with $10 \mathrm{~mm}$-EDTA of washed bacteria from the $100 \mathrm{ml}$ cultures was as previously described (Poxton \& Byrne, 1981) except that the dialysis step was omitted after it was shown to be unnecessary.

Preparation of antisera. Antisera to C. novyi type A NCTC 538 and C. sporogenes NCTC 8594 were raised in New Zealand White rabbits by intravenous injection of whole, washed UV-killed bacteria over a 5 week period as detailed by Poxton (1979). A standard dose of $10^{9}$ organisms per injection was used. These sera are referred to as reference species antisera.

Indirect enzyme-linked immunosorbent assay (ELISA). The EDTA extracts were diluted to $30 \mu \mathrm{g}$ protein $\mathrm{ml}^{-1}$ in antigen buffer ( $50 \mathrm{mM}$-sodium carbonate buffer, $\mathrm{pH} 9.6)$ and $50 \mu \mathrm{l}$ volumes were used to coat wells in flatbottomed polystyrene microtitre plates. They were incubated for $4 \mathrm{~h}$ at $37^{\circ} \mathrm{C}$ and overnight at $4{ }^{\circ} \mathrm{C}$. After washing three times with $0.15 \mathrm{M}-\mathrm{NaCl}$ containing $0.05 \%(\mathrm{v} / \mathrm{v})$ Tween 20 (Tween saline), doubling dilutions ( 1 in 400 to 1 in 102400 ) of rabbit antiserum in Tween PBS (50 mM-sodium phosphate buffer pH 7.4 containing $0.15 \mathrm{M}-\mathrm{NaCl}$, $0.05 \%$ Tween 20 and $0.02 \%$ sodium azide) were added to each well $(50 \mu \mathrm{l})$ and incubated for $4 \mathrm{~h}$ at room temperature. The plates were washed three times in Tween saline, and anti-rabbit IgG-alkaline phosphatase conjugate (Miles, Slough, UK) diluted 1 in 500 in Tween PBS was added to each well $(50 \mu \mathrm{l})$ and incubated overnight at room temperature. After washing three times in Tween saline, $50 \mu 1 p$-nitrophenyl phosphate (Sigma) at a concentration of $1 \mathrm{mg} \mathrm{ml}^{-1}$ in $50 \mathrm{~mm}$-sodium carbonate buffer $\mathrm{pH} 9 \cdot 8$, containing $1 \mathrm{mM}-\mathrm{MgCl}_{2}$, was added to each well. After incubation for $1 \mathrm{~h}$ at room temperature, the plates were read in a Titertek Multiskan (Organon Teknika, St Neots, UK): the highest dilution of serum that gave an $A_{405}>1.0$ was recorded as the titre.

Polyacrylamide gel electrophoresis and electroblot transfer. SDS-polyacrylamide gel electrophoresis was done on $10 \%$ slab gels with the buffer system of Laemmli (1970) as described by Poxton \& Brown (1979). Approximately $50 \mu \mathrm{g}$ protein (based on the Lowry method) was run in each track.

Electroblot transfer was essentially by the method of Towbin et al. (1979). Material was transferred to nitrocellulose membrane (Transblot Transfer Medium, Bio-Rad) in Tris/glycine/methanol buffer, pH $8 \cdot 3$ over a $16 \mathrm{~h}$ period at $12 \mathrm{~V}, 40 \mathrm{~mA}$. The transferred antigens were detected with the ImmunBlot immunoassay (Bio-Rad). Briefly, the membrane, after washing for $10 \mathrm{~min}$ in Tris-buffered saline ( $20 \mathrm{~mm}$-Tris, $500 \mathrm{mM}-\mathrm{NaCl}, \mathrm{pH} 7 \cdot 5$; TBS) was placed in $3 \%(\mathrm{w} / \mathrm{v})$ gelatin TBS for $45 \mathrm{~min}$ to block any unbound sites. It was then transferred into the $C$. sporogenes or $C$. novyi antiserum diluted 1 in 200 in $1 \%$ gelatin TBS and incubated for $3 \mathrm{~h}$ at room temperature. After two $10 \mathrm{~min}$ washes in $0.025 \%$ Tween 20 TBS the membrane was placed into goat anti-rabbit IgG-horseradish peroxidase conjugate (Bio-Rad) diluted 1 in 3000 in $1 \%$ gelatin TBS and incubated for $1 \mathrm{~h}$ at room temperature. After a further two 10 min washes in Tween TBS it was placed into HRP colour development solution (Bio-Rad) which contains 4-chloro-1-napthol and hydrogen peroxide. Colour developed within 5-15 min. The reaction was stopped by placing into distilled water and washing with several changes of water over $1 \mathrm{~h}$. All of the above steps were done in clean glassware and with gentle agitation throughout.

\section{RESULTS}

\section{ELISA studies}

EDTA extracts of all of the test strains were reacted in an ELISA system with our reference antisera to $C$. sporogenes and $C$. novyi (Table 1). All the proteolytic type B, three out of four of the type $\mathrm{A}$ and one type $\mathrm{E}$ strains reacted strongly with the $C$. sporogenes reference species 
Table 1. Reactions obtained with EDTA extracts of C. botulinum, $C$. sporogenes and $C$. novyi in ELISA with antisera to $C$. sporogenes and C. novyi

The figures presented are typical of results obtained in repeated experiments. --, Titre of less than 400 .

\begin{tabular}{|c|c|c|}
\hline $\begin{array}{l}\text { EDTA antigen } \\
\text { prepared from: }\end{array}$ & \multicolumn{2}{|c|}{$\overbrace{\text { C. sporogenes NCTC } 8594 \text { C. novyi NCT }}$} \\
\hline \multicolumn{3}{|l|}{ C. botulinum } \\
\hline Al & 1600 & - \\
\hline A7 7 & 3200 & - \\
\hline A8 & 400 & - \\
\hline A9 & - & - \\
\hline $\mathrm{pB} 25$ & 6400 & - \\
\hline $\mathrm{pB} 26$ & 400 & - \\
\hline $\mathrm{pB} 27$ & 800 & - \\
\hline npB 188 & - & - \\
\hline npB194 & - & - \\
\hline npB229 & - & - \\
\hline C205 & - & 1600 \\
\hline D254 & - & 3200 \\
\hline E34 & - & - \\
\hline E118 & 6400 & - \\
\hline E126 & - & - \\
\hline E250 & - & - \\
\hline \multicolumn{3}{|l|}{ C. sporogenes } \\
\hline NCTC 8494 & 6400 & - \\
\hline 24 & 1600 & - \\
\hline 33 & 1600 & - \\
\hline \multicolumn{3}{|l|}{ C. novvi } \\
\hline A NCTC 538 & - & 12800 \\
\hline A GR2A & - & 800 \\
\hline A GR3A & - & 1600 \\
\hline B GR2B & - & 1600 \\
\hline C NCTC 9747 & - & 1600 \\
\hline D NCTC 8350 & - & 1600 \\
\hline
\end{tabular}

antiserum. The type A strain that did not react (A9) was not even a weak reaction; no colour was detectable by the ELISA plate reader at the lowest dilution ( 1 in 400$)$ used. The two heterologous $C$. sporogenes strains reacted significantly with the reference $C$. sporogenes antiserum. No reaction was demonstrated between the non-proteolytic $B$, the $C$, the $D$, three out of four of the $E$ strains and all of the $C$. novyi strains with the $C$. sporogenes antiserum.

With the $C$. novyi antiserum only $C$. novyi strains and $C$. botulinum strains of types $C$ and D showed a reaction.

\section{Polyacrylamide gel electrophoresis and electroblot transfer}

Initially, EDTA extracts from one strain of each type of $C$. botulinum and from one strain each of $C$. sporogenes and $C$. novyi were run on $10 \%$ SDS-PAGE slab gels and stained with Coomassie blue (Fig. 1a). All patterns were complex but there were marked similarities between those of $C$. botulinum types A and B (proteolytic) and those of types C and D (tracks 2 and 3, and 5 and 6 respectively).

Two duplicates of this gel (unstained) were electrophoretically transferred to nitrocellulose membranes and were probed with the $C$. sporogenes and $C$. novyi antisera. The results, after development with the horseradish peroxidase conjugate reagents, are shown in Fig. $1(b, c)$. The antiserum to $C$. sporogenes (Fig. 1 b) reacted significantly with $C$. botulinum type Al (track 2 ) and proteolytic type B25 (track 3 ) and the homologous $C$. sporogenes (track 8 ) EDTA extracts. In the cross-reaction with the $C$. botulinum proteolytic type B25 extract many strongly staining bands were visible, the overall intensity being stronger than with the homologous reaction. The cross- 



Fig. 1. SDS-polyacrylamide gel electrophoresis and corresponding electroblot transfers of EDTA extracts of strains of $C$. botulinum, $C$. novyi and $C$. sporogenes. Approximately $50 \mu \mathrm{g}$ protein in $50 \mu \mathrm{l}$ sample buffer were run on each track of a $10 \%$ acrylamide gel. Track 1, C. novyi type A NCTC 538; track 2, C. botulinum A1; track 3,C. botulinum pB25; track 4, C. botulinum npB188; track 5, C. botulinum C205; track 6, C. botulinum D154; track 7, C. botulinum E34; track 8, C. sporogenes NCTC 8594. (a) Coomassie blue-stained gel. $(b)$ Electroblot transfer of $(a)$ reacted with antiserum to $C$. sporogenes NCTC 8594. (c) Electroblot transfer of $(a)$ reacted with antiserum to $C$. novyi NCTC 538.

reaction with the type A extract was less strong but several bands, which were common to the type B, were stained. Single faint cross-reacting bands were also apparent in the $C$. novyi and the C. botulinum non-proteolytic type B extracts. The antiserum to C. novyi (Fig. 1 c) only crossreacted with extracts from $C$. botulinum types $\mathrm{C}$ and $\mathrm{D}$, one major antigen being present in each. 


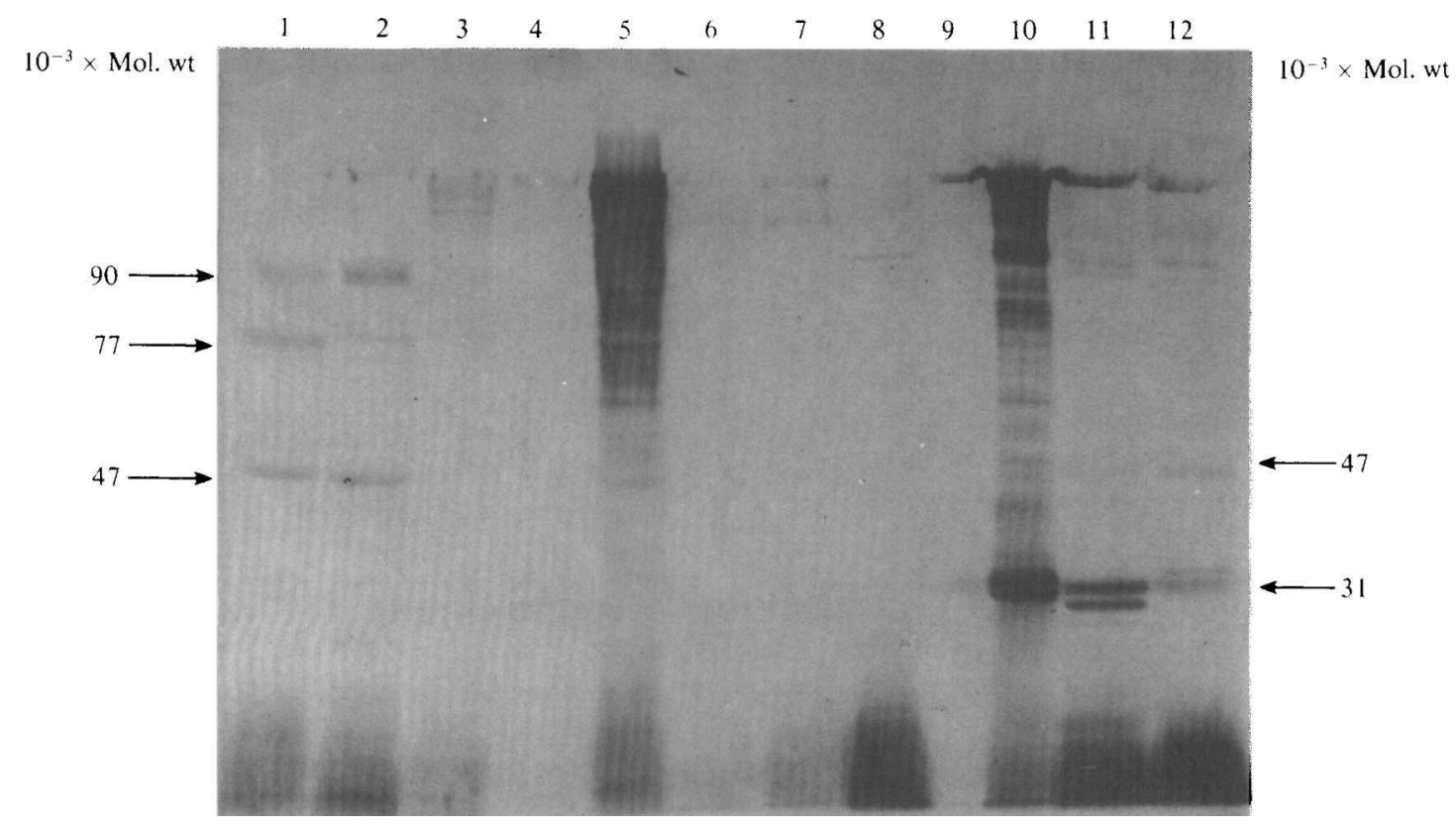

Fig. 2. Electroblot transfers of EDTA extracts of strains of $C$. botulinum and $C$. sporogenes separated as in Fig. $1(a)$ and reacted with antiserum to $C$. sporogenes NCTC 8594. Track 1, C. botulinum A1; track 2, C. botulinum A7; track 3, C. botulinum A8; track 4, C. botulinum A9; track 5, C. botulinum pB25; track 6, C. botulinum pB26; track 7, C. botulinum pB27; track 8, C. botulinum E118; track 9, blank; track 10, $C$. sporogenes NCTC 8594; track 11, C. sporogenes 24; track 12, C. sporogenes 33.

In order to demonstrate fully the cross-reactions revealed by ELISA between $C$. botulinum strains and the two antisera and also to compare these inter-species cross-reactions with the reactions within $C$. sporogenes and $C$. novyi strains, two further electroblot transfers were done. EDTA extracts of all the strains of type A and proteolytic B, strain E118 (see Table 1) of $C$. botulinum and the three $C$. sporogenes strains were run together on an SDS polyacrylamide gel. They were transferred to nitrocellulose and reacted with the $C$. sporogenes antiserum (Fig. 2). Extracts from A1 and A7 (tracks 1 and 2) showed almost identical patterns with a series of diffusely staining bands at the bottom of the gel and three common bands at molecular weights of approximately 90000,77000 and 47000 . The A8 extract (track 3) was similar to A1 and A7 except that the bands were weaker in staining intensity and some high molecular weight bands were faintly visible at the top of the gel. The proteolytic type B strains (tracks 5-7) produced a much more variable reaction than the type A strains. The extract from B25 (track 5) produced a very complex pattern of strongly staining bands as already described. Reactions with extracts from B26 and B27 were much weaker but the patterns produced were similar to each other. A significant reaction was also produced by the extract from strain E118 (track 8). A series of strongly staining low molecular weight bands at the front of the gel was the main feature of this pattern with a few faint higher molecular weight bands. When compared with the homologous reaction (track 10) the two other strains of $C$. sporogenes produced much less complex patterns (tracks 11 and 12). Again the series of low molecular weight bands was common, while there were other common bands with molecular weight of approximately 47000 and 31000 .

Similarly the type $C$ and $\mathrm{D}$ strains of $C$. botulinum and all the $C$. novyi strains were run together and reacted with the $C$. novyi type $\mathrm{A}$ antiserum (Fig. 3). The $C$. botulinum types $\mathrm{C}$ and $\mathrm{D}$ (tracks 1 and 2) both produced similar patterns. A band with a molecular weight of approximately 47000 was the major cross-reacting antigen, but several high molecular weight bands were apparent at the top of the gel and there was a series of regularly spaced cross-reacting low molecular weight bands at the bottom of the gel. The reactions between the three type A C. novyi strains were very 




Fig. 3. Electroblot transfers of EDTA extracts of strains of $C$. botulinum and $C$. novyi separated as in Fig. $1(a)$ and reacted with antiserum to $C$. novyi type A NCTC 538. Track 1, C. botulinum C205; track 2, C. botulinum D154; track 3, blank; track 4, C. novyi A NCTC 538; track 5, C. novyi A GR2A; track 6, C. novyi A GR3A; track 7, C. novyi B GR2B; track 8, C. novyi C NCTC 9747; track 9, C. novyi D NCTC 8350.

similar to each other (tracks 4, 5 and 6). The major cross-reacting antigens had molecular weights of 48000 and 31000 with a series of regularly spaced bands at the bottom of the gel. The C. novyi types B, C and D produced similar patterns (tracks 7,8 and 9 ): there was a major band with a molecular weight of 47000 and a series at the bottom of the gel which was similar to all the other tracks on the gel.

\section{DISCUSSION}

The results obtained with the ELISA and the electroblot procedures agree closely and show conclusively that most strains of $C$. botulinum types A and proteolytic B (Group I) share antigens with $C$. sporogenes, whereas types $C$ and D (Group III) share antigens with $C$. novyi. This provides further evidence of the close taxonomic relationships between these species. In view of the limited number of strains investigated, it is not yet certain how widely these cross-reactions occur and how many exceptions there may be to the general rule demonstrated here. For example, one of the strains of $C$. botulinum type A did not react, whilst one of the type E strains did react with $C$. sporogenes antiserum. Moreover, varying degrees of cross-reactivity could be demonstrated with the three type A and three proteolytic type B strains that cross-reacted with the $C$. sporogenes antiserum. This was also true for the three strains of $C$. sporogenes investigated, and indicates antigenic heterogeneity. Reactions within the $C$. novyi strains were much more uniform. No major differences were seen between the three $C$. novyi type A strains. The $C$. novyi strains of types B, C and D showed close antigenic similarity with each other and with the $C$. botulinum strains of types $\mathrm{C}$ and $\mathrm{D}$.

The patterns of cross-reactivity revealed by electroblot transfer all showed some similarities. Most of the major cross-reactive bands corresponded to predominant protein bands on the Coomassie blue-stained gels. All strains that reacted with antiserum, however, also showed 
patterns of regularly spaced bands at the bottom of the gels. These bands did not appear to correspond to any of the protein bands on the Coomassie blue-stained gels. They were reminiscent of the patterns produced by lipopolysaccharides of Gram-negative bacteria when stained with silver (Tsai \& Frasch, 1982). It is possible that these cross-reacting antigens were lipocarbohydrate molecules from the cytoplasmic membrane (lipoteichoic acid analogues). We know that, at least for $C$. difficile and $C$. sordelli, the membrane lipocarbohydrates are crossreacting antigens and that they are solubilized by EDTA treatment (Poxton \& Cartmill, 1982).

There was no attempt made in this study to use antiserum raised to any $C$. botulinum strain. The aim was to use antisera raised against cells that were as close as possible to living organisms: washing and killing with a minimum dose of UV light were the only treatments used. Obtaining undenatured cells of toxigenic $C$. botulinum devoid of toxin is not possible. No attempt was made to work with $C$. botulinum types $\mathrm{F}$ and $\mathrm{G}$ as the author was not protected against these types.

This study was funded by a grant from the Biomedical Research Committee of the Scottish Home and Health Department (grant no. K/MRS/50/C247). I am extremely grateful to Dr J. S. Crowther and Mr C. Grimes of Unilever Research, Sharnbrook, Bedfordshire for the advice, assistance and facilities made available during my visit to their laboratory. Professor J. G. Collee's interest and encouragement is gratefully acknowledged.

\section{REFERENCES}

EkLund, M. W. \& Poysky, F. T. (1981). Relationships of bacteriophages to the toxigenicity of Clostridium botulinum and closely related organisms. In Biomedical Aspects of Botulism, pp. 93-109. Edited by G. E. Lewis. New York: Academic Press.

Hatheway, C. L. \& McCroskey, L. M. (1981). Laboratory investigation of human and animal botulism. In Biomedical Aspects of Botulism, pp. 165180. Edited by G. E. Lewis. New York: Academic Press.

Holbrook, W. P., Duerden, B. I. \& Deacon, A. G. (1977). The classification of Bacteroides melaninogenicus and related species. Journal of Applied Bacteriology 42, 259-273.

Holdeman, L. V., Cato, E. P. \& Moore, W. E. C. (editors) (1977). Anaerobe Laboratory Manual, 4th edn. Blacksburg, Va., USA: Anaerobe Laboratory, Virginia Polytechnic Institute and State University.

Iida, H., Oguma, K. \& Inoue, K. (1981). Toxin production and phage in Clostridium botulinum types C and D. In Biomedical Aspects of Botulism, p. 119. Edited by G. E. Lewis. New York: Academic Press.

Kiritani, K., Mitsui, S., NaKamura, S. \& Nishida, S. (1973). Numerical taxonomy of Clostridium botulinum and Clostridium sporogenes strains, and their susceptibilities to induced lysins and to mitomycin C. Japanese Journal of Microbiology 17, 361-372.

LAEMMLI, U. K. (1970). Cleavage of structural proteins during the assembly of the head of bacteriophage T4. Nature, London 277, 680-685.

Poxton, I. R. (1979). Serological identification of Bacteroides species by an enzyme-linked immunosorbent assay. Journal of Clinical Pathology 32, 294-298.

Poxton, I. R. \& Brown, R. (1979). Sodium dodecyl sulphate-polyacrylamide gel-electrophoresis of cell- surface proteins as an aid to the identification of the Bacteroides fragilis group. Journal of General Microbiology 112, 211-217.

Poxton, I. R. \& BYRNe, M. D. (1981). Immunological analysis of the EDTA-soluble antigens of Clostridium difficile and related species. Journal of General Microbiology 122, 41-46.

PoXTON, I. R. \& BYRne, M. D. (1984). The demonstration of shared antigens in the genus Clostridium by an enzyme-linked immunosorbent assay. Journal of Medical Microbiology (in the Press).

Poxton, I. R. \& CarTmill, T. D. I. (1982). Immunochemistry of the cell-surface carbohydrate antigens of Clostridium difficile. Journal of General Microbiology 128, 1365-1370.

SMITH, L. D. S. (1977). The Organism. In Botulism. The Organisms, its Toxins, the Disease, pp. 15-33. Springfield, Ill., USA: Thomas.

Towbin, H., Staehlin, T. \& Gordon, J. (1979). Electrophoretic transfer of proteins from polyacrylamide gels to nitrocellulose sheets: procedure and some applications. Proceedings of the National Academy of Sciences of the United States of America 76, $4350-4354$.

TSAI, C.-M. \& Frasch, C. E. (1982). A sensitive silver stain for detecting lipopolysaccharides in polyacrylamide gels. Analytical Biochemistry 119, 115 119.

W ALKER, P. D. \& BATTY, I. (1964). Fluorescent studies in the genus Clostridium. II. A rapid method for differentiating Clostridium botulinum types A, B and F, types C and D, and type E. Journal of Applied Bacteriology 27, 140-142.

Willis, A. T. (1977). Anaerobic Bacteriology: Clinical and Laboratory Practice, 3rd edn. London: Butterworths. 